\title{
Book Reviews -
}

\section{Atlas of Chest Imaging Correlated Anatomy With MRI and CT. By Marvin Wagner and Thomas L. Lawson. New York: Raven Press, 1992, 134 pp.}

This book is designed to supplement the authors' previous text on Segmental Anatomy: Application of Clinical Medicine published in 1982. In that text, the authors did not include any MR and studied the entire body. In the current volume, the authors include MR and CT and specifically limit their efforts to the chest. The book is designed to "provide medical students, surgeons, radiologists, and all practitioners in medicine a detailed, photographic map of segmental anatomy." Anatomical illustrations have all been harvested from segmental anatomy. The book is organized into three units. The first is axial views, the second parasagittal and sagittal views, and the third coronal views. There are no color illustrations. The atlas of 134 pages includes an index of only two pages. Bibliography takes up less than one page, containing eight references.

This book meets its objective, detailing the anatomical pictures with CT and MR. In the first section of axial images, it is a pity that the anatomical images are so much larger than the radiographic ones. The anatomical picture is not always facing the corresponding imaging picture. Instead, there may be one or two anatomical pictures and then one or two images. All sections have precise labeling of exactly where the plane of section is. The CT and MR pictures are of good quality, if not of the most up-todate images.

An omission, however, lies in the few references and in the subject index, which does not list such things as the aortic-pulmonary window or refers to a figure which shows this. For CT anatomy of the lungs it is important to show secondary lobules, and there is not even a reference to such in the index.

This book is nicely done with adequate pictures. It deserves a place in the libraries of those individuals or departments doing cross-sectional thoracic imaging. It is hoped that in the next edition more attention will be paid to clinical areas of concern and list those in the index.

-Thomas L. SLovis, mD

Department of Radiology

Wayne State University School of Medicine

Children's Hospital of Michigan

Detroit, Michigan
The Lung in Rheumatic Diseases. Vol. 45 of Lung Biology in Health and Diseases. Executive Editor: Claude Lenfant. Editors: Grant W. Cannon, Guy A. Zimmerman. New York: Marcel Dekker, Inc., 1992, 545 pp.

The Lung in Rheumatic Diseases is a textbook discussing the various pulmonary abnormalities that occur with connective tissue diseases. The book is divided into 4 sections comprising a total of 17 chapters. Twenty-six authors with expertise in pulmonary and rheumatic diseases contributed in writing this book. The 4 sections cover lung pathology in joint diseases, structural and functional alterations in lung disease, pulmonary involvement with rheumatic diseases, and multisystem disorders involving the lung and joints. Each chapter is easy to read and provides excellent references. The pathologic and radiologic pictures are clear, and most of the tables easy to follow. A broad range of topics is covered in a concise manner.

Although The Lung in Rheumatic Diseases is not specifically directed toward the pediatric pulmonologist, I found it to be a good reference that addresses numerous topics relevant to pulmonologists caring for children with pulmonary complications secondary to rheumatic diseases. Several chapters included data and briefly discussed pediatric lung abnormalities in connective tissue diseases. However, the book was written with the differential diagnoses and therapies directed toward adult care. Chapters covering broad topics, such as pathophysiology and mechanisms of lung injury, are well written and relevant to both adult and pediatric practice.

Section I provides a review of the general mechanisms involved in lung injury, highlighting the development of alveolitis and pulmonary fibrosis. Chapter 2 of Section I considers the role of the pleura in connective tissue diseases. Pleural effusions, their etiology, and the pathophysiologic processes involved in development of effusions are examined. A synopsis of pleural effusions in rheumatoid arthritis systems, lupus erythematosus, and scleroderma is given.

Section II consists of 4 chapters reviewing lung function and structure in rheumatologic diseases. Tests utilized in diagnosing pulmonary diseases are reviewed, including lung biopsies, fiberoptic bronchoscopy, and various radiologic imaging techniques. The usefulness of these and other tests is discussed in relationship to rheumatologic lung diseases. Chapter 6 summarizes the 